\title{
Properties of light scalar mesons from lattice QCD.
}

\author{
C. McNeile ${ }^{1}$ and C. Michael ${ }^{1}$ \\ (UKQCD Collaboration) \\ ${ }^{1}$ Theoretical Physics Division, Dept. Math. Sci., \\ University of Liverpool, Liverpool L69 7ZL, UK.
}

\begin{abstract}
Lattice QCD with $N_{f}=2$ flavours of sea quark is used to explore the spectrum and decay of scalar mesons. We are able to determine the $b_{1}-a_{0}$ mass difference and this leads to the conclusion that the lightest non-singlet scalar meson $\left(a_{0}\right)$ has a mass of $1.01(4) \mathrm{GeV}$. We determine from the lattice the coupling strength to KK and $\pi \eta$. We compute the leptonic decay constant of the lightest non-singlet scalar meson. We discuss the impact of these lattice results on the interpretation of the $a_{0}(980)$ state. We also discuss $K_{0}^{*}$ states.
\end{abstract}

PACS numbers: 12.38.Gc, 14.40.Cs, 13.25.-k

\section{INTRODUCTION}

The scalar mesons known experimentally do not fit into a tidy pattern, as found for vector or axial mesons, for example. Because the scalar mesons have S-wave decays to light two-body states (two pseudoscalar mesons), then the impact of these two-body channels on the scalar meson can be sizeable. Thus the scalar mesons may have $\bar{q} \bar{q} q$ as well as $\bar{q} q$ components. For example, there are two $a_{0}$ mesons, $a_{0}(980)$ and $a_{0}(1450)$, known 1]. The $a_{0}(980)$ meson is closely associated with the $\bar{K} K$ threshold and it has been suggested that this is a molecular state. This is can be explored using lattice techniques. A further complication is that the flavour singlet scalar mesons can mix with scalar glueballs, although here we restrict our investigation to the flavour non-singlet scalar mesons from lattice QCD.

There has been a long history of studying the scalar non-singlet mesons on the lattice. These states tend to have a poorer signal to noise ratio than the S-wave mesons 2] such as the $\rho$ and $\pi$, hence are less commonly studied. Much of the early literature on light $\mathrm{P}$-wave mesons focussed on designing good interpolating operators to create the mesons [2, 3].

The quenched studies of the $a_{0}$ were complicated by the discovery of a ghost state that made the correlator for the $a_{0}$ particle, which should be positive definite in a unitary quantum field theory, go negative [4]. If this effect was not taken into account then the chiral extrapolation of correlators was unreliable. Modern studies of this state such as those by Burch et al. 5] correct for the effect of the missing contribution to the $0^{++}$correlator from the $\eta^{\prime}$ meson. Prelovsek [6] has also studied the ghost state in the $a_{0}$ correlator using $2+1$ dynamical staggered fermions and mixed (chiral valence and staggered sea) fermions. In both cases, which are essentially partially quenched, deviant features are discovered.

The non-singlet scalar mass is an input into the study of mixing with glueballs in the singlet sector by Weingarten and Lee [7]. The ghost state was not taken into account and this led to problems with the chiral extrapolation of the non-singlet $0^{++}$meson masses. This mixing

\begin{tabular}{c|c|c} 
Group & Method & $m_{a_{0}} \mathrm{GeV}$ \\
\hline Bardeen at al. [4] & quenched & $1.34(9)$ \\
Hart et al. [9] & $n_{f}=2$, partially quenched, & $1.0(2)$ \\
Prelovsek et al. [12] & $n_{f}=2$, unquenched, & $1.58(34)$ \\
Prelovsek et al. [12] & partially quenched & $1.51(19)$ \\
Burch et al. [5] & quenched & $\sim 1.45$ \\
\hline
\end{tabular}

TABLE I: Some results for the mass of the $a_{0}$ meson from quenched and partially quenched QCD that include the effect of the ghost state [4].

has also been discussed 8, 9] using unquenched lattices which avoids this problem.

Alford and Jaffe [10] used quenched QCD with $\bar{q}^{2} q^{2}$ operators relevant to $0^{++}$mesons. Their study claimed to see evidence for bound states in the $\bar{q}^{2} q^{2}$ channel relevant to $0^{++}$states. The work of Alford and Jaffe [10] can be criticised for not taking into account the quenched ghost in the $a_{0}$ correlator. Only a subset of the correlators required for the singlet channel were computed. This is, perhaps, consistent in quenched QCD but clearly important physics is omitted.

The scalar collaboration are starting to use lattice QCD techniques to study the $\kappa$ particle [11].

Prelovsek et al. [12] extended the work of Bardeen et al. 4] on the effect of the ghost state in the $a_{0}$ channel to the partially quenched theory. By restricting lattice study to valence quarks heavier than the sea-quarks, Hart et al. 9] were able to extrapolate to light quarks with no ghost contributions, obtaining an estimate for the $a_{0}$ mass of $1.0(2) \mathrm{GeV}$.

In table \we collect together some recent numbers for the mass of the $a_{0}$ mass from some modern lattice calculations that take into account the ghost term. None of the calculations in table \ had complete control over all systematic errors, such as finite size effects or the continuum limit, even within quenched QCD. The results for the lightest $0^{++}$meson are mostly around $1.5 \mathrm{GeV}$. As we note above, the $a_{0}$ decays via the strong interaction, so a quenched QCD calculation may give a poor estimate of the particle mass. 
The MILC collaboration reported evidence for $a_{0}$ decay on the lattice in an unquenched lattice QCD calculation with $2+1$ flavours of improved staggered fermions with a lattice spacing of $0.12 \mathrm{fm}$ [13]. In MILC's first paper they found the $a_{0}$ mass to be significantly lower than the mass of the $b_{1}$ and $a_{1}$ mesons. This was different behaviour from the quenched study with the same parameters. MILC 13] found that, for lighter quarks, the mass of the $a_{0}$ meson was close to the sum of the $\pi$ and $\eta$ masses, where the mass of the $\eta$ was estimated using the Gell-Mann-Okubo formula. As the MILC collaboration [14] ran unquenched calculations with even lighter sea quarks they confirmed that the lightest state in the $a_{0}$ channel lay below the $\pi \eta$ threshold. Using independent techniques on a subset of the configurations from MILC, Gregory et al. [15] also found that the lightest state in the $a_{0}$ channel was below the $\pi \eta$ threshold. Prelovsek [6] has studied $a_{0}$ decay using staggered chiral perturbation theory, concluding that taste violations in the staggered fermion formalism allow a small amplitude for the decay of the $a_{0}$ state to two pions. The decay $a_{0} \rightarrow \pi \pi$ is forbidden in the real world because of $G$ parity.

Since the current state of lattice investigation of scalar mesons is incomplete, more work is needed. In order to make a start in establishing the nature of scalar mesons from first principle in $\mathrm{QCD}$, we address here the flavour non-singlet scalar mesons. As is well known, lattice QCD in the quenched approximation is not a consistent theory and this manifests itself as ghost contributions to the scalar meson propagation - arising from the spurious lowlying threshold in the $\pi \eta$ two-body channel. We use here $N_{f}=2$ dynamical gauge configurations so that we have a consistent field theory. The physical case, however, also has another light quark (the $s$ quark) and has lighter $u$ and $d$ quarks than we are able to use on a lattice. Thus some extrapolation will be needed to obtain consequences for the physical spectrum.

As we approach the limit of physical light quark masses, the scalar mesons become unstable: they are resonances. On dynamical lattices these decay channels are open. Thus we need to have methods to cope with unstable particles on a lattice. The study of hadronic decays from the lattice is not straightforward - see ref. [16]. It is possible, however, to evaluate the appropriate hadronic matrix element from a lattice if the transition is approximately on-shell. This allows us to estimate decay widths, provided that the underlying coupling is relatively insensitive to the quark masses. We follow methods generically similar to those used by us to study $\rho$ decay 17] and hybrid meson decay [18].

As well as the hadronic decay, one can also define a decay constant analogously to that defined for the weak decay of pseudoscalar mesons. We discuss the relevance of this and the determination from the lattice of the scalar decay constant.

\section{SPECTRUM}

As a by-product of our study of hybrid mesons, we have accurate lattice measurements of the $a_{0}, b_{1}$ and $a_{1}$ mesons from clover-improved lattices with $N_{f}=2$ degenerate sea-quarks - see Table II and Table III for details. Each of these mesons is unstable and in the $N_{f}=2$ world with two degenerate quarks they have two-body decays to $\pi \eta_{2}, \pi \omega$ and $\pi \rho$ respectively. Here $\eta_{2}$ is flavour singlet, $(\bar{u} u+\bar{d} d) / \sqrt{2}$, so it is more like the $\eta^{\prime}$ than the $\eta$ meson. Indeed estimates 21] of its mass from a mixture of lattice results and experiment suggest that it is near $0.86 \mathrm{GeV}$ for light quarks of physical mass. Thus, for these light quarks with $N_{f}=2$, the open decay channel is heavier for the $a_{0}$ meson than for the $a_{1}$ and $b_{1}$ mesons. Hence, in the self-consistent world with $N_{f}=2$ degenerate light quarks, we do not expect the $a_{0}$ meson to have any peculiar features compared to the other P-wave mesons.

This is in contrast to quenched QCD where the flavour singlet pseudoscalar has the same mass as the pion, but an anomalous coupling. Moreover, quenched QCD allows a contribution (hairpin diagram) to the $a_{0}$ correlator from this two-body channel which gives significant unphysical effects.

The conventional way to extract the mass of a meson is to use lattice simulations at successively smaller quark masses and to extrapolate using an expression based on chiral perturbation theory. For dynamical simulations, which are mandatory here, one has a very limited range of quark mass available. Resorting to partially quenched methods to reach lighter valence quarks is potentially dangerous, if the valence quarks are lighter than the sea quarks. Indeed a study using partially quenched methods on the U355 and U350 data sets has been conducted 9] and yielded an estimate of the $a_{0}$ mass of 1.0(2) GeV. Here we explore a more reliable way to obtain the $a_{0}$ meson mass.

Since the decay channels open to the P-wave mesons are quite similar, we propose to focus on the mass differences between them since this will reduce lattice artifacts. The $a_{1}$ meson is very wide, experimentally, so that it is not a good point of comparison with lattice results. The $b_{1}$ meson, however is relatively narrow and should be well reproduced on a lattice. Indeed in ref. 18], we were able to measure from the lattice the decay amplitude for the S-wave decay $b_{1} \rightarrow \pi \omega$, obtaining agreement with experiment. For lattice U355, for example,the decay threshold is at $0.72(4)$ for $a_{0}$ from $\pi \eta_{2}$ and at $0.883(8)$ for $b_{1}$ from $\pi \omega$, in lattice units. These energy values are both above the mass values we report in Table III] so each state is stable on our lattice and they are about equally below the lowest threshold.

We show our results from two state fits to a $2 \times 3$ matrix of correlators $(2 \times 2$ for U350 and C390) using $t$-range 3-12 (3-10 for C390 and C410) in Table TII The methods used are described in more detail in ref. [18]. We use local and extended sources at the source (and two sizes of extension at the sink in some cases). The 
excited mass values are in all cases significantly higher (by over 50\%) than the ground state values reported and do not correspond to any simple two-body level. Thus the $\pi \eta_{2}$ threshold level at $a E=0.72$ for U355 does not feature in the fit. As we shall see later, this is consistent with the relatively weak transition amplitude on a lattice between two-body states and the $a_{0}$.

We find that the $a_{0}$ correlator can have big fluctuations which are apparent at large $t$, most noticeably for U350 where the zero-momentum effective mass decreases at large $t$. The origin of these fluctuations is mixing between the $a_{0}$ and the pion induced by regions of oddparity in the vacuum - presumably associated with instantons. See [22] for a discussion of $a_{0}-\pi$ mixing in lattice QCD and in the instanton liquid model. With sufficient statistics these odd-parity fluctuations average to zero. Using stochastic methods (all-to-all) helps to reduce these fluctuations as we reported before [18]. Using non-zero momentum can also act as a useful cross-check. This suggests that for U350 with zero momentum, we should use a $t$-range from 3-8 to reduce these fluctuation effects and retain consistency with our results from momentum $2 \pi n / L$ where $n=(1,0,0)$ and $(1,1,0)$. The value for U350 quoted in Table III is from this analysis.

For the $b_{1}$ meson, at non-zero momentum there can be mixing with the $\rho$ meson (for some spin states). For the non-local (fuzzed) operators there will also be an admixture of $L=2$ (from distortion due to Lorentz boost) and possibly some mixing of opposite $\mathrm{C}$ (unless the momentum phase factors are applied symmetrically to the fuzzed operator). For these reasons we rely on zero momentum for the $b_{1}$ meson.

Since the $b_{1}$ meson has an unambiguous interpretation as predominately a bound state of a quark and antiquark, we show our spectrum results for it versus quark mass in fig [1] Here we see that our lattice results are quite consistent with a smooth extrapolation to the experimental mass value for physical light quarks. To have a precision determination of the mass would require a continuum extrapolation as well as an extrpolation in quark mass and we do not have data sufficient to undertake this combined extrapolation.

Because of the difficulties in extrapolating to light quarks using lattice results with a range of different lattice spacings, we focus on mass differences. Here we concentrate on the difference $m\left(b_{1}\right)-m\left(a_{0}\right)$ which is plotted against the quark mass in fig. 2] using $r_{0}$ determined on the lattices to create a dimensionless comparison.

The point in fig. 2 labelled $a_{0}(980)$ assumes that the relevant $a_{0}$ meson is the lightest with mass $984.7 \mathrm{MeV}$. The next heaviest with mass $1474 \mathrm{MeV}$ is less well established and would correspond to a point (-0.66) far below the $x$-axis. Our lattice results for the mass difference show no significant dependence on the quark mass, and averaging our lattice results gives an estimate (using $r_{0}=0.5 \mathrm{fm}$ ) of $m\left(b_{1}\right)-m\left(a_{0}\right)=221(40) \mathrm{MeV}$. There is an additional systematic error coming from the assumption of a constant difference as the quark mass is

\begin{tabular}{lllllll} 
Code no. $\kappa$ & $m(\pi) r_{0}$ & $r_{0} / a$ & $a m(\pi)$ & $a m(\rho)$ \\
\hline C410 & 237 & 0.1410 & 1.29 & 3.01 & $0.427(1)$ & $0.734(4)$ \\
C390 & 648 & 0.1390 & 1.93 & 2.65 & $0.729(1)$ & $0.969(2)$ \\
U355 200 & 0.1355 & 1.47 & 5.04 & $0.292(2)$ & $0.491(7)$ \\
U350 151 & 0.1350 & 1.93 & 4.75 & $0.405(5)$ & $0.579(8)$
\end{tabular}

TABLE II: Lattice gauge configurations U355 and U350 from UKQCD 23] and C390 and C410 from CP-PACS 24] are used, all having spatial extent $\mathrm{L}=16 \mathrm{a}$. These have $N_{f}=2$ flavours of sea quark and we use valence quarks of the same mass as the sea quarks.

$$
\begin{array}{llll}
\text { Code } a m\left(b_{1}\right) & a m\left(a_{1}\right) & a m\left(a_{0}\right) \\
\hline \text { C410 } & 1.17(3) & 1.15(2) & 1.03(4) \\
\text { C390 } & 1.48(4) & 1.39(5) & 1.33(8) \\
\text { U355 } & 0.77(2) & 0.72(2) & 0.64(4) \\
\text { U350 } & 0.87(2) & 0.88(2) & 0.75(3)
\end{array}
$$

TABLE III: Results for P-wave mesons from the methods of ref. [18] for U355 and C410 and from conventional methods for U350 (with 4 time sources) and C390.

decreased, which we are unable to quantify. As discussed above, our lattice masses in Table III are at quark masses around the strange quark mass and at non-zero lattice spacing. They correspond, as expected, to masses somewhat larger than the physical $b_{1}$ mass of $1230 \mathrm{MeV}$, but are consistent within the expected systematic errors of the extrapolations necessary.

As discussed above, we do not expect the two-body thresholds to play a significant role in our $N_{f}=2$ spectra. We do, however, measure these decay transitions to have a more complete analysis.

\section{HADRONIC DECAYS}

For the case of $N_{f}=2$ degenerate quarks, the matrix elements for decay transitions of a non-singlet scalar meson to two pseudo-scalar mesons are given in Table IV where the quark diagrams are illustrated in fig. 3.

Only one case, $a_{0} \rightarrow \pi \eta_{2}$, is allowed staying strictly within $N_{f}=2$ with valence quarks of the same properties as sea quarks (here $\eta_{2}$ is the flavour singlet pseudoscalar for $N_{f}=2$, namely $\left.(\bar{u} u+\bar{d} d) / \sqrt{2}\right)$. This case involves a disconnected diagram (D) and is not directly relevant to phenomenology. In the limit that the strange quark is much heavier than the $u$ and $d$ quarks, we expected the neglect of $s$ quarks in the sea to be a good approximation. In that case, decays such as $K_{0}^{*} \rightarrow K \pi$ can be studied from diagram $T$. For the physical case with $s$ quarks of some $80 \mathrm{MeV}, a_{0} \rightarrow \pi \eta_{8}$ and $a_{0} \rightarrow \bar{K} K$ may also be determined adequately from $N_{f}=2$ lattice study of diagram $T$.

With this in mind, we first evaluate the lattice transition amplitude corresponding to the connected triangle diagram $T$. The contribution of $T$ to various decay 


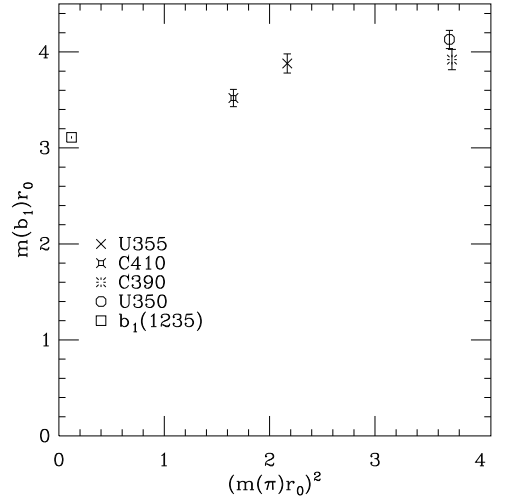

FIG. 1: Mass of the $b_{1}$ mesons (in units of $r_{0} \approx 0.5 \mathrm{fm}$ ) versus quark mass. The strange quark mass corresponds to $\left(m(\pi) r_{0}\right)^{2} \approx 3.4$.

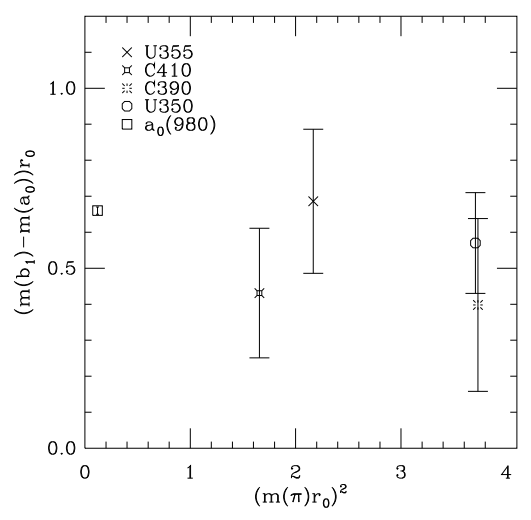

FIG. 2: Mass difference of $b_{1}$ and $a_{0}$ mesons (in units of $r_{0} \approx 0.5 \mathrm{fm}$ ) versus quark mass.

amplitudes will have the numerical factors listed in Table IV The most relevant cases will be $a_{0} \rightarrow K \bar{K}$ and $K^{*} \rightarrow K \pi$. This is a partially-quenched evaluation in the sense that we use valence $s$-quarks (of the same mass as our $u, d$ sea-quarks) which are not present in the sea. We are able to use similar methods to those used to study $\rho$ decay [17] and hybrid meson decay [18].

The lattice results for the connected $(T)$ contribution to a generic scalar meson transition to two pseudoscalar mesons are presented as the normalised lattice ratio

$$
R(t)=\frac{\left(S \rightarrow P_{1} P_{2}\right)}{\sqrt{(S \rightarrow S)\left(P_{1} \rightarrow P_{1}\right)\left(P_{2} \rightarrow P_{2}\right)}}
$$

where the three-point correlator is constructed from

\begin{tabular}{lllll}
$\mathrm{S}$ & $P_{1}$ & $P_{2}$ & $T$ & $D$ \\
\hline$a_{0}$ & $\pi$ & $\eta_{2}$ & $2^{1 / 2}$ & $-2^{1 / 2}$ \\
$a_{0}$ & $\mathrm{~K}$ & $\overline{\mathrm{K}}$ & 1 & 0 \\
$a_{0}$ & $\pi$ & $\eta_{s s}$ & 0 & -1 \\
$a_{0}$ & $\pi$ & $\eta_{8}$ & $(2 / 3)^{1 / 2}$ & 0 \\
$\mathrm{~K}^{*}$ & $\mathrm{~K}^{+}$ & $\pi^{0}$ & $2^{-1 / 2}$ & 0 \\
$\mathrm{~K}^{*}$ & $\mathrm{~K}^{0}$ & $\pi^{+}$ & 1 & 0 \\
$\mathrm{~K}^{*}$ & $\mathrm{~K}$ & $\eta_{2}$ & $2^{-1 / 2}$ & $-2^{-1 / 2}$ \\
$\mathrm{~K}^{*}$ & $\mathrm{~K}$ & $\eta_{s s}$ & 1 & -1
\end{tabular}

TABLE IV: Coefficients of transition amplitudes from flavour non-singlet scalar meson $\mathrm{S}$ to $P_{1} P_{2}$ for the triangle quark diagram $(T)$ and the disconnected quark diagram $(D)$. Only the top line is allowed if $N_{f}=2$ strictly. The other lines are allowed when a valence $s$ quark is added. We define $\eta_{2}$ as $(\bar{u} u+\bar{d} d) / \sqrt{2}, \eta_{s s}$ as $\bar{s} s$ and $\eta_{8}$ as $(\bar{u} u+\bar{d} d-2 \bar{s} s) / \sqrt{6}$. We have assumed that the disconnected contributions to the decay to $\eta_{8}$ cancel.
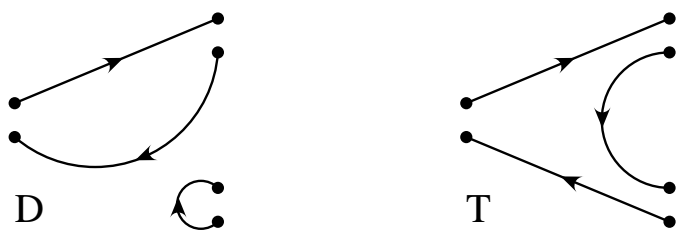

FIG. 3: Quark diagrams involved in the decays listed in Table IV where $D$ is the disconnected diagram and $T$ is the triangle diagram.

propagators as illustrated for $T$ in fig. 3. Each two and three-point correlator is taken at the same time separation $t$.

Since the $a_{0}$ mass is approximately twice the pseudoscalar mass (see Table III) at zero momentum, we have an on-shell transition and we expect [17, 18] the ratio $R(t)$ to be approximately linear with slope $x a$ versus $t$ where $x$ is the lattice transition amplitude. This is indeed observed, as shown in fig. fig:xta0.

We first checked that using different operators to create mesons gave essentially the same ratio $R(t)$. We use local or fuzzed operators for each of the three particles involved and in each case the ratio is the same within errors for the $t$ region of interest for the case we studied in most detail, namely with all momenta zero.

The most reliable determination of the coupling constant comes from using meson operators which minimise excited state contributions. We use fuzzing with separations of $3 a(\mathrm{C} 410)$ or $5 a$ (U355) to achieve this. We extract the slope $x a$ by taking finite differences and relate this lattice transition amplitude to the continuum coupling via Fermi's Golden Rule. The derivation of the phase space factor is described in ref. 19]. Then, to compare different lattice data sets, we extract the effective coupling using [17, 18, 20]

$$
g^{2}=\frac{1}{\pi}(x a)^{2}(L / a)^{3} \frac{a E\left(P_{1}\right) E\left(P_{2}\right)}{E\left(P_{1}\right)+E\left(P_{2}\right)}
$$




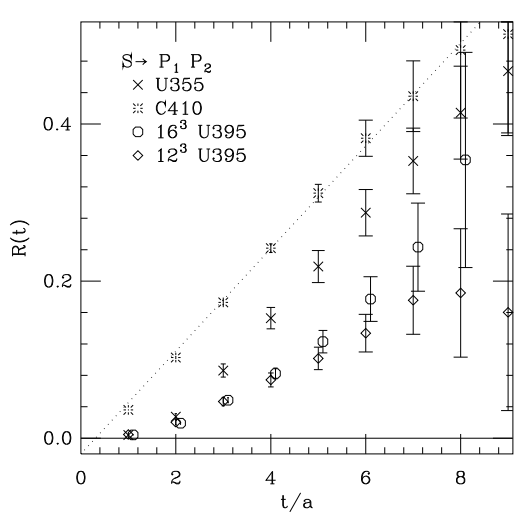

FIG. 4: The normalised ratio $R(t)$ for the connected contribution $(T)$ to the transition $S \rightarrow P_{1} P_{2}$. The contribution of $T$ to particular decays can be read off from Table IV The number of lattice gauge configurations analysed was 90 (U355), 165 (C410) and 30 for each U395 case. The dotted line illustrates the expected behaviour with slope $x a$ for C410.

Here the decay width $\Gamma$ is, for a process with amplitude $T$, given by $\Gamma / k=g^{2}$, where $k$ is the centre of mass momentum of the decay products. For particular transitions, the quark coupling coefficients of Table IV also enter, squared, in the decay rate.

As a first check of this approach, we evaluated the effective coupling from lattices that differ only in spatial size (labelled U395, see ref. [18] for more details) and we found excellent agreement when the spatial volume was changed by a factor of 2.4., as shown in fig. 目

The coupling extracted, as above, from our higher statistics data-sets is shown in fig. 5 This shows a coupling $g \approx 1$ which has implications which we discuss later. The consistency between the two determinations (C410 and U355) which have different spatial volumes and different lattice spacings is satisfactory. As an overall summary we quote a coupling $g=1.0(2)$.

We also have available some results (from 40 gauge configurations for U355 and for 50 for C410) for transitions involving non-zero momentum, especially $S(1) \rightarrow$ $P_{1}(0)+P_{2}(1)$ where the momentum (in units of $2 \pi / L$ ) is given in the brackets. These results used the methods of ref. 17, 18 respectively. The normalised lattice ratio $R(t)$ is shown in fig. [6] and the coupling extracted assuming the formulae above is included for C410 (where we used an optimum method to extract ground state contributions) in fig. (4 As discussed in ref. 17], the decay in flight poses some problems of normalisation (since it is not quite equivalent to a centre of mass decay with relative momentum $\pi / L$ ), so must have a somewhat bigger systematic error to compensate. Nevertheless, we see an approximate agreement of the lattice transition amplitude $x a$ and of the coupling $g$ when the decay has

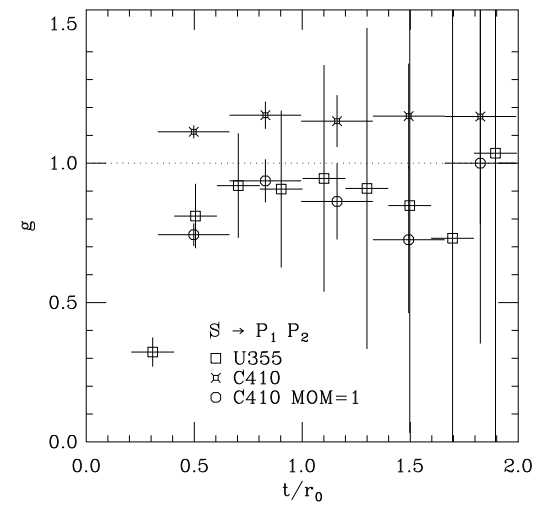

FIG. 5: The effective coupling $g$ extracted from $R(t)$ as described in the text for the triangle graph $T$ for $S \rightarrow P_{1} P_{2}$ with zero momentum (also some results for non-zero momentum as discussed in the text). The dotted line at $g=1$ is to guide the eye.

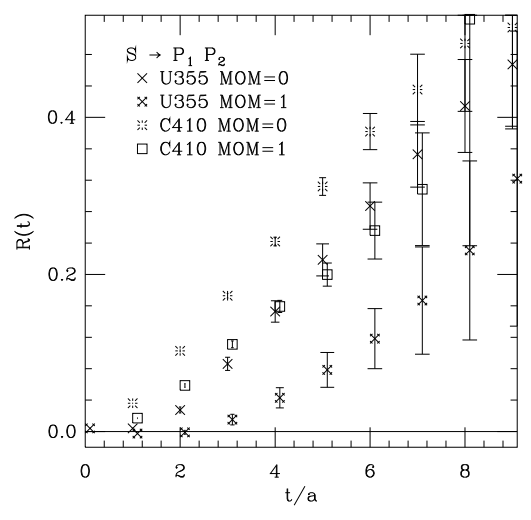

FIG. 6: The normalised ratio $R(t)$. Here $\mathrm{MOM}=0,1$ refers to the transition $S(k) \rightarrow P_{1}(0)+P_{2}(k)$ with momenta $k=2 n \pi / L$ with $n=0$ and 1 .

momentum release of zero and of $\pi / L$. This is to be expected for an S-wave decay where the effective matrix element should be independent of momentum.

\section{LEPTONIC DECAY CONSTANT}

The decay constants of non-singlet $0^{++}$mesons are not routinely calculated using lattice QCD, although they are of interest for a number of reasons. The value of the decay constant, which is basically the amplitude to find 
a quark and anti-quark at the origin, can help distinguish between different quark content of the meson 25, 26]. For instance, if the $a_{0}$ was a $\bar{K} K$ molecule, then the decay constant would be small relative to the value of the pion decay constant. The decay constant of the $a_{0}$ meson is also a theoretical input to study of $\mathrm{B}$ meson decays and of $\tau$ decays to final states that include an $a_{0}[27,28,29$, , 30].

Diehl and Hiller discuss the prospects of determining the value of the decay constant of the $a_{0}$ mesons from experiment 27. As we explain below, a direct measurement of the decay $a_{0}$ constant coupled with computation of a QCD matrix element could be used to compute the mass difference of the up and down quarks.

The decay constant of the light $0^{+}$meson can be defined by equation 1

$$
\left\langle 0\left|V_{\mu}^{a b}\right| a_{0}\right\rangle=i p_{\mu} g_{a_{0}}
$$

where $V_{\mu}^{a b}$ is the vector current for quark flavours $a$ and $b$.

The conservation of the vector current is used to relate the operator in equation 1 to the scalar current.

$$
\partial_{\mu}\left(\overline{q^{a}} \gamma_{\mu} q^{b}\right)=i\left(m_{a}-m_{b}\right) \overline{q^{a}} q^{b}
$$

for light quarks with flavour $a$ and $b$. This motivates a definition of the decay constant such as

$$
i\left\langle 0\left|\overline{q^{u}} q^{d}\right| a_{0}\right\rangle=\hat{f}_{a_{0}} m_{a_{0}}^{2}
$$

To compare the size of the decay constant of the $a_{0}$ to that of the $K^{*}(1430)$ meson, Maltman [25] defined a new decay constant with a slightly different normalisation.

The direct use of equation 2 is impossible in a lattice calculation with two degenerate flavours of sea quarks. The vector current does not couple to the scalar meson in this case. The decay constant in equation [3] is nonzero in an unquenched lattice QCD calculation with two flavours of sea quarks.

There is another reason for splitting the definition of the $0^{++}$meson decay constant into a quark mass factor and QCD matrix element. Currently there is a disagreement between the value of the strange quark from unquenched lattice QCD calculations that use different types of fermion for the light quarks [31]. Some recent papers 32, 33, 34] report summaries of the values for the strange quark mass published around time of the lattice 2005 conference, using different formulations of lattice QCD. Lattice QCD calculations are only just starting to report values for the differences between the masses of the up and down quarks [35]. Hence, we prefer to quote separately our measured matrix element rather than introduce explicit factors of the quark mass. So, it is more natural to define the decay constant using equation 4

$$
\left\langle 0|\bar{q} q| a_{0}\right\rangle=m_{a_{0}} f_{a_{0}}
$$

The relation between $f_{a_{0}}$ and $g_{a_{0}}$ is via

$$
g_{a_{0}}=\frac{m_{d}-m_{u}}{m_{a_{0}}} f_{a_{0}}
$$

\begin{tabular}{ccc|c}
$\kappa$ & $\mathrm{a} f_{a_{0}} / \hat{Z}_{S}$ & $\hat{Z}_{S}$ & $f_{a_{0}} \mathrm{MeV}$ \\
\hline .1355 & $0.352(19)$ & 0.70 & $488(26)$ \\
.1350 & $0.346(30)$ & 0.71 & $460(40)$ \\
.1410 & $0.474(48)$ & 0.79 & $478(48)$ \\
.1390 & $0.480(97)$ & 0.84 & $513(104)$ \\
\hline
\end{tabular}

TABLE V: Our results for decay constant of the $a_{0}$ meson.

The explicit factor of the quark masses $\left(m_{d}-m_{u}\right)$ in equation [5] is the reason that Narison [36] computes the value of $g_{a_{0}}$ to be between 1.3 and $1.6 \mathrm{MeV}$.

The matrix element in equation 4 is extracted from the amplitudes in the fits to the correlators (see [37] for example). The raw numbers from the lattice calculation need renormalisation. To convert the lattice number to the $\overline{M S}$ scheme we use tadpole improved perturbation theory to one loop order [38]. The renormalisation factor for a scalar current, at the scale $\mu=1 / a$, is

$$
Z_{S}(\mu=1 / a)=u_{0}\left(1-\alpha_{s} S_{c}\right)
$$

where $u_{0}$ is the fourth root of the plaquette, and the constant $S_{c}$ is 1.002 for the Wilson gauge action [39] and 0.5031 for the Iwasaki gauge action [40,41].

To remove $O(a)$ terms we also need to use improvement coefficients. We define the renormalisation $\hat{Z}_{S}$ that includes the improvement factor

$$
\hat{Z}_{S}=Z_{S}\left(1+b_{S} m_{q}\right)
$$

where $m_{q}$ is the mass of the light quark. We used the one loop expression for $b_{S}$.

$$
b_{S}=\left(1+\alpha_{s} b_{s c}\right)
$$

where the constant $b_{s c}$ is 1.3722 for the Wilson action [42], and 1.2800 for the Iwasaki action [41, 43]. We used the coupling computed in the $\overline{M S}$ scheme. For the UKQCD data set we used the coupling determined on the same data set 44]. For the CP-PACS data we used the $\overline{M S}$ coupling quoted in their paper 24]. The coupling was evaluated at the scale $\mu=1 / a$. Our results are in table $\nabla$ As we only have decay constants for two different quark masses with the same action, we do not attempt a chiral extrapolation. The dependence of the decay constant on the pion mass seems small, however. In table $\mathbf{\nabla 1}$ we compare our results to other determinations of the decay constants. The results in table $\nabla$ show that the decay constant $f_{a_{0}}$ is not suppressed relative to the pion decay constant.

The decay constant of the $0^{++}$meson is one of the parameters in the model that gets rid of the ghost state in the scalar $0^{++}$correlator in quenched QCD [4] and partially-quenched QCD 12], so there are estimates for it. These studies of the $0^{++}$used another normalisation convention for the scalar decay constant, so we do not tabulate their values here.

Chernyak 45] uses a fit to data with a factorisation assumption to obtain $g_{0^{+}}=70 \pm 10 \mathrm{MeV}$ for the $K^{*}(1430)$. 


\begin{tabular}{c|c|c} 
Group & Method & $f_{a_{0}} \mathrm{MeV}$ \\
\hline Maltman [25] & sum rule & 298 \\
Shakin and Wang. [46] & model & 433 \\
Narison. [36] & sum rule & $320-390$ \\
\hline
\end{tabular}

TABLE VI: Some results for decay constant of the $a_{0}$ meson. We used a value of $m_{d}-m_{u}$ of $4 \mathrm{MeV}$ to convert the normalisation of Narison's estimate. The quark masses quoted by Shakin and Wang were used to convert normalisation conventions for other two results.

Converting to our normalisation conventions, using a nominal value of the strange quark mass of $100 \mathrm{MeV}$, this corresponds to $f_{K^{*}(1430)}=1000 \pm 140 \mathrm{MeV}$. The results for the decay constants in table $\nabla$ are larger than the results of UKQCD's recent calculation of the decay constant of the $0^{+}$charm-light meson [37].

As an aside we note that if the decay constant $g_{0+}$ of the $a_{0}$ or $K^{\star}(1430)$ was measured experimentally, then it would allow an additional method to measure the quark mass differences $m_{u}-m_{d}, m_{s}-m_{d}$ respectively, using lattice estimates of the QCD matrix elements.

\section{DISCUSSION}

For the non-strange flavour non-singlet scalar meson $\left(a_{0}\right)$, our lattice determinations using the self-consistent $N_{f}=2$ approximation to QCD give clear support for a physical $a_{0}$ meson lying substantially lighter than the $b_{1}$. The mass estimates we find are consistent with the observed $a_{0}$ at $950 \mathrm{MeV}$ but not the heavier state at 1474 $\mathrm{MeV}$.

To relate our approach to experiment with an additional strange quark, we can assume that the strange quark pair production is relatively small and so can be neglected. For $\mathrm{K}_{0}^{*}$ propagation, for example, this amounts to treating the $\mathrm{K} \pi$ channel correctly but having an anomalous contribution from $\mathrm{K} \eta_{s s}$ intermediate states. Here the $\eta_{s s}$ propagation has a missing piece (just as $\eta_{2}$ does in the $\eta_{2} \pi$ contribution to the $a_{0}$ propagation in quenched QCD with $N_{f}=2$ ) and so will not have a single exponential but two contributions with masses corresponding to (i) the connected pseudoscalar meson with valence quarks of strange mass and (ii) the $\eta_{2}$ meson. Both of these contributions are not especially light, so we do not expect any major distortion of the $\mathrm{K}_{0}^{*}$ from using valence $s$-quarks. Similarly the $a_{0}$ decays to $K \bar{K}$ and $\eta_{8} \pi$ are expected to be accessible without major distortion from the neglect of strange quarks in the sea, as we discussed above.

For the strange scalar mesons, the $\mathrm{K}_{0}^{*}(1430)$ with mass $1412 \mathrm{MeV}$ is heavier than the corresponding axial mesons ( $\mathrm{K}_{1}^{*}$ with masses 1273 and $1402 \mathrm{MeV}$ ). These two axial mesons are related to a mixture of the strange partners of the non-strange $b_{1}$ and $a_{1}$ mesons since charge conjugation is not a good quantum number for strange mesons.
So the interpretation in this case is unclear. As well as this strange scalar meson at $1412 \mathrm{MeV}$, one might expect a lighter state, about $100-130 \mathrm{MeV}$ heavier (mass split determined from tensor mesons) than the $a_{0}(980)$. The so-called kappa $(\kappa)$ at $700-900 \mathrm{MeV}$ with a very broad width (400 MeV or more) has been claimed by many sources [1] and a recent analysis [47] gives mass $750_{-50}^{+30}$ $\mathrm{MeV}$. There is no consensus yet on the existence of the kappa, because some analyses of experimental data see no sign of it [4]. Our lattice studies suggest that a scalar $\mathrm{K}_{0}^{*}$ meson of mass around 1000-1200 MeV would be expected in a theory with $N_{f}=2$ sea quarks and a strange quark treated as a valence quark. For our case where the valence $s$-quark and $u, d$ sea-quarks have the same mass, the anomalous $K \eta_{s s}$ intermediate state combines with the $K \pi$ intermediate state to give only a $K \eta_{2}$ intermediate state, just like the case of $a_{0}$ propagation. Hence our lattice treatment does not correctly include the $K \pi$ threshold in the $\mathrm{K}_{0}^{*}$ meson propagation and so may be less reliable than the $a_{0}$ propagation.

The connected decay diagram $(T)$ is appropriate for the decays $a_{0} \rightarrow K K, a_{0} \rightarrow \pi \eta_{8}$ and $K_{0} \rightarrow K \pi$ where the appropriate factors are given in Table IV Then the experimental data [1] can be used to estimate the coupling (from $\Gamma / k$ ). For $\mathrm{K}_{0}^{*}(1430)$, this gives $g^{2}=0.32(3)$. While for the $\kappa$, one recent analysis 47 finds a width of $342 \pm 60 \mathrm{MeV}$ which corresponds to $g^{2}=0.7(2)$. For $a_{0}(980)$, the state is close to the $\bar{K} K$ threshold which distorts the appearance of the meson. Phenomenological analyses vary but one example quotes [49] a total width of $153 \mathrm{MeV}$ and a coupling given by $g^{2}=0.82$ for $\bar{K} K$ and around 0.7 for $\eta \pi$. For $a_{0}(1450)$, the partial widths are not well known and one can only estimate that the $\bar{K} K$ and $\eta \pi$ decays yield couplings smaller than $g^{2}=0.23$ and 0.34 respectively.

Our determination of the coupling which controls decay is also relevant for identification of states. We find a coupling (normalised to diagram $T$ above) given by $g \approx 1$. This favours the lighter $a_{0}$ and $\kappa$ meson over the heavier states. Our determination of the $a_{0}$ decay constant disfavours a molecular structure for this state, in agreement with our conclusion from hadronic decays. The only concern is that for the $K_{0}^{*}$ meson the experimental evidence gives a $\kappa$ meson lighter than our expectations.

\section{CONCLUSION}

We have studied the spectrum and decay of non-singlet scalar mesons from first principles using lattices with a consistent (unitary) field theoretic interpretation for $N_{f}=2$ flavours of sea-quark. Rather than extrapolate the scalar masses directly, we concentrate on the mass splitting between the $a_{0}$ and $b_{1}$ mesons from the lattice. The lattice results are unambiguous and point to a scalar meson which is 221(40) MeV lighter than the $b_{1}$. Since the experimental mass value of the $b_{1}$ meson is $1230 \mathrm{MeV}$, this suggests that the $a_{0}(980)$ is indeed the lightest non- 
singlet scalar meson in a theory with $N_{f}=2$ flavours of degenerate quark. Our approach does not include the $K \bar{K}$ channel, so this channel is to be regarded as having an impact on a pre-existing state, rather than as being the dominant component of the state. In other words, we do not find that a $K \bar{K}$ molecule is a good approximation to the $a_{0}(980)$.

Our results for the decay transition amplitude are also consistent with the phenomenological estimates of the coupling of the $a_{0}(980)$ to $K \bar{K}$ and $\eta \pi$. Overall, we conclude that the $a_{0}(980)$ is predominantly a conventional meson with normal couplings to $\bar{q} q$.

For the $K_{0}^{*}$ scalar meson, we expect a mass 100-130 $\mathrm{MeV}$ heavier than the $a_{0}$ (based, for example, on the observed mass splittings of the tensor mesons). This is not easily related to any experimental candidate: the $\kappa$ is too light $(700-900 \mathrm{MeV})$, while the $K_{0}^{*}(1430)$ is too heavy. What may help clarification is that we find a decay coupling transition (to $K \pi$ ) which is comparable to that needed phenomenologically for the $\kappa$ but much larger than that needed for the $K_{0}^{*}(1430)$. This suggests that the $\kappa$ is more closely related to the state obtained in $N_{f}=2$ lattice QCD. A lattice treatment with the strange quark included in the sea would help to clarify further this conclusion.

\section{ACKNOWLEDGEMENTS}

The authors acknowledge support from PPARC grant PPA/Y/S/2003/00176. This work has been supported in part by the EU Integrated Infrastructure Initiative Hadron Physics (I3HP) under contract RII3-CT-2004506078. We acknowledge the ULGrid project of the University of Liverpool for making available computer resources. We acknowledge the CP-PACS collaboration [24] for making available their gauge configurations.
[1] Particle Data Group, S. Eidelman et al., Phys. Lett. B592, 1 (2004),

[2] T. A. DeGrand and M. W. Hecht, Phys. Rev. D46, 3937 (1992), hep-lat/9206011

[3] UKQCD, P. Lacock, C. Michael, P. Boyle, and P. Rowland, Phys. Rev. D54, 6997 (1996), hep-lat/9605025

[4] W. A. Bardeen, A. Duncan, E. Eichten, N. Isgur, and H. Thacker, Phys. Rev. D65, 014509 (2002), hep-lat/0106008

[5] T. Burch et al., (2006), hep-lat/0601026

[6] S. Prelovsek, Phys. Rev. D73, 014506 (2006), hep-lat/0510080

[7] W.-J. Lee and D. Weingarten, Phys. Rev. D61, 014015 (2000), hep-lat/9910008

[8] UKQCD, C. McNeile and C. Michael, Phys. Rev. D63, 114503 (2001), hep-lat/0010019

[9] UKQCD, A. Hart, C. McNeile, and C. Michael, Nucl. Phys. Proc. Suppl. 119, 266 (2003), hep-lat/0209063

[10] M. G. Alford and R. L. Jaffe, Nucl. Phys. B578, 367 (2000), hep-lat/0001023

[11] Scalar, T. Kunihiro et al., PoS LAT2005, 034 (2005),

[12] S. Prelovsek, C. Dawson, T. Izubuchi, K. Orginos, and A. Soni, Phys. Rev. D70, 094503 (2004), hep-lat/0407037

[13] C. W. Bernard et al., Phys. Rev. D64, 054506 (2001), hep-lat/0104002

[14] C. Aubin et al., Phys. Rev. D70, 094505 (2004), hep-lat/0402030

[15] E. B. Gregory, A. C. Irving, C. McNeile, S. Miller, and Z. Sroczynski, PoS LAT2005, 027 (2005), hep-lat/0510066

[16] C. Michael, PoS LAT2005, 008 (2005), hep-lat/0509023

[17] UKQCD, C. McNeile and C. Michael, Phys. Lett. B556, 177 (2003), hep-lat/0212020

[18] UKQCD, C. McNeile and C. Michael, (2006), hep-lat/0603007

[19] UKQCD,C. McNeile et al., Phys. Rev. D65, 094505 (2002), hep-lat/0201006
[20] C. Michael, Nucl. Phys. Proc. Suppl. 128, 153 (2004), hep-lat/0310010

[21] UKQCD, C. R. Allton et al., Phys. Rev. D70, 014501 (2004), hep-lat/0403007

[22] P. Faccioli and T. A. DeGrand, Phys. Rev. Lett. 91, 182001 (2003), hep-ph/0304219

[23] UKQCD, C. R. Allton et al., Phys. Rev. D65, 054502 (2002), hep-lat/0107021

[24] CP-PACS, A. Ali Khan et al., Phys. Rev. D65, 054505 (2002), hep-lat/0105015

[25] K. Maltman, Phys. Lett. B462, 14 (1999), hep-ph/9906267

[26] S. Narison, (2005), hep-ph/0512256

[27] m. diehl and g. hiller, jhep 06, 067 (2001), hep-ph/0105194

[28] BABAR, B. Aubert et al., Phys. Rev. D70, 111102 (2004), hep-ex/0407013.

[29] BABAR, F. Couderc, (2005), hep-ex/0506031

[30] D. Delepine, J. L. Lucio M., and C. A. Ramirez, Eur. Phys. J. C45, 693 (2006), hep-ph/0501022

[31] P. E. L. Rakow, Nucl. Phys. Proc. Suppl. 140, 34 (2005), hep-lat/0411036

[32] ALPHA, M. Della Morte et al., Nucl. Phys. B729, 117 (2005), hep-lat/0507035

[33] M. Gockeler et al., (2006), hep-lat/0601004.

[34] HPQCD, Q. Mason, H. D. Trottier, R. Horgan, C. T. H. Davies, and G. P. Lepage, (2005), hep-ph/0511160

[35] MILC, C. Aubin et al., Phys. Rev. D70, 114501 (2004), hep-lat/0407028

[36] S. Narison, Phys. Lett. B216, 191 (1989),

[37] UKQCD, G. Herdoiza, C. McNeile, and C. Michael, (2006), hep-lat/0604001

[38] G. P. Lepage and P. B. Mackenzie, Phys. Rev. D48, 2250 (1993), hep-lat/9209022

[39] T. Bhattacharya, R. Gupta, W.-J. Lee, and S. R. Sharpe, Phys. Rev. D63, 074505 (2001), hep-lat/0009038

[40] Y. Taniguchi and A. Ukawa, Phys. Rev. D58, 114503 (1998), hep-lat/9806015

[41] S. Aoki, K.-i. Nagai, Y. Taniguchi, and A. Ukawa, Phys. 
Rev. D58, 074505 (1998), hep-lat/9802034

[42] S. Sint and P. Weisz, Nucl. Phys. Proc. Suppl. 63, 856 (1998), hep-lat/9709096

[43] S. Aoki, R. Frezzotti, and P. Weisz, Nucl. Phys. B540, 501 (1999), hep-lat/9808007

[44] QCDSF-UKQCD, S. Booth et al., Phys. Lett. B519, 229 (2001), hep-lat/0103023

[45] V. Chernyak, Phys. Lett. B509, 273 (2001), hep-ph/0102217
[46] C. M. Shakin and H. Wang, Phys. Rev. D63, 074017 (2001),

[47] D. V. Bugg, Phys. Lett. B632, 471 (2006), hep-ex/0510019

[48] S. N. Cherry and M. R. Pennington, Nucl. Phys. A688, 823 (2001), hep-ph/0005208

[49] N. N. Achasov and A. V. Kiselev, Phys. Rev. D68, 014006 (2003), hep-ph/0212153. 